\title{
Extraction of Anthocyanin from Eggplant Peel by Ultrasonic Assisted Method and Application in Cosmetics
}

\author{
Xiaofen $\mathrm{Fu}^{1, \dagger}$, Chunli Zhu ${ }^{1, \dagger}{ }^{\dagger}$, Ao Shen ${ }^{1}$, Weixi Fan ${ }^{1}$, Chaoyue Shan ${ }^{1}$ \\ ${ }^{1}$ School of Chemistry and Biological Engineering, Qilu Institute of Technology, Jinan, 250200, P. R. China. \\ ${ }^{\dagger}$ These two authors contribute equally to this work.
}

\begin{abstract}
The anthocyanin of eggplant skin was extracted by ultrasonic assisted method. Single factor experiment and orthogonal optimization experiment were used to optimize the extraction factors of anthocyanin from eggplant peel. The results showed that the optimum extraction of anthocyanin from eggplant peel was obtained by orthogonal experiment under the condition of feed-liquid ratio of 13:15, time of $40 \mathrm{~min}$, and temperature of $50^{\circ} \mathrm{C}$. The anthocyanin is stable by treating $1 \mathrm{~min}$ after $100^{\circ} \mathrm{C}$.
\end{abstract}

\section{Introduction}

Anthocyanin (Anthocyanin), also known as anthocyanins, are water-soluble flavonoids found in the epidermis of flowers, fruits and stems and leaves of plants and in the cortical cells of the following table, especially in dark red, blue or purple fruits. The color of anthocyanin can change with the acidity and alkalinity of cell fluid, the acidity of cell fluid is red, and the alkalinity of cell fluid is blue. Anthocyanin has the ability of anti-oxidation, antimutation and so on, which plays an important role in human physiological function [1-3]. Therefore, it is of great significance to reduce the cost of anthocyanin extraction. Although the synthetic pigment has bright color, good stability and low price, natural anthocyanins have been paid more and more attention in food, medicine and cosmetics because of its toxicity [4-5].

Eggplant (Solano melongena L.) is an annual herb. The shape of the fruit is long or round. The color is mostly purple or purple black, less white, red and so on. Eggplant tastes delicious, and it is nutritious and popular. Eggplant peel is rich in anthocyanins and has certain development value as natural food pigment.

At present, anthocyanin extraction methods include ultrasonic extraction, microwave extraction, organic solvent extraction and so on. Because organic solvents are mostly toxic reagents, they are volatile and have low extraction rate, and they are faced with organic solvent residues, which have a bad effect on human health. Therefore, the selection of non-toxic organic solvent for eggplant skin anthocyanin extraction has positive practical significance.

\section{Materials and methods}

\subsection{Materials}

Eggplant, distilled water, anhydrous ethanol, glucose, phosphate buffer, UV-Vis spectrophotometer, (7600CRT, Shanghai jinghua technology Co.,Ltd) ultrasonic cleaner (Sinobakr,) traditional Chinese medicine pulverizer(DF50-A,dade), constant temperature water tank(BHS-1,LAB FISH), electronic balance(shanghai jinghua technology Co., Ltd), electrothermalconstant temperature blast dryer, Abbe refractometer.(DigPol-R200,Shanghai jiahang technology Co.,Ltd)

\subsection{Extraction methods}

\subsubsection{Pretreatment}

The eggplant was deviated, separated pericarp and pulp. Then the eggplant peel was washed and dried, and cut into small pieces in electrothermal blast dryer. The small pieces of eggplant peel were baked to constant weight at $60^{\circ} \mathrm{C}$, and crushed to powder. Finally the powder of eggplant peel was sealed storage and set aside.

\subsubsection{Anthocyanins}

In this experiment, ultrasonic assisted ethanol extraction method can accelerate the destruction of plant cell wall, improve the extraction rate of intracellular substances and shorten the extraction time [6].

A $25 \mathrm{~g}$ pretreated powder was added to a round bottom flask containing a certain amount of anhydrous ethanol, and then rocked and heated for a certain time. The

*Corresponding author: Chunli Zhu; e-mail: chunli0125@163.com. 
ultrasonic extracted extract was transferred to the centrifuge tube and the supernatant was centrifuged and separated. Since the extraction rate of anthocyanin is positively correlated with the absorbance at wavelength $525 \mathrm{~nm}$, the extraction rate of anthocyanin can be indirectly reflected by the determination of absorbance at $525 \mathrm{~nm}$.

\subsubsection{Process flow}

eggplant peel $\rightarrow$ dry $\rightarrow$ smash $\rightarrow$ sieving $\rightarrow$ ultrasonic extraction $\rightarrow$ centrifuge $\rightarrow$ keep liquid supernatant $\rightarrow$ take samples of the liquid supernatan and measure absorbancy $\rightarrow$ calculate the extuaction rate

\subsection{Single-factor experiment}

\subsubsection{Time on extraction rate of anthocyanins}

The extraction rate of anthocyanin in different ultrasonic extraction time was determined at a wavelength of $525 \mathrm{~nm}$. at a solid-liquid ratio of 1:15 and an extraction temperature of $50^{\circ} \mathrm{C}$, with the same concentration of glucose aqueous solution as a blank control, and 10 min were selected as the unit time interval of $10-50 \mathrm{~min}$. The results were determined three times parallelly.

\subsubsection{Temperature on extraction rate of anthocyanins}

The extraction rate of anthocyanin in different ultrasonic extraction temperature was determined at a wavelength of $525 \mathrm{~nm}$ 、 at a solid-liquid ratio of 1:15 and the highest extraction rate of time, with the same concentration of glucose aqueous solution was added as blank control. And $10^{\circ} \mathrm{C}$ was selected as the unit time interval of $20-60^{\circ} \mathrm{C}$. The results were determined three times parallelly.

\subsubsection{Solid-liquid ratio on extraction rate of anthocyanins}

The solid-liquid ratio refers to the ratio of eggplant powder to anhydrous ethanol. At the wavelength of $525 \mathrm{~nm}$, the extraction temperature of $50^{\circ} \mathrm{C}$, and the extraction time of $40 \mathrm{~min}$, the extraction rate of anthocyanins at different solid-liquid ratios was determined. The solid-liquid ratios were $1: 5,1: 7.5,1: 10,1: 12.5,1: 15,1: 17.5,1: 20,1: 22.5$, $1: 25$. The results were determined three times parallelly.

\subsection{Thermal stability of anthocyanins}

Thermal stability of anthocyanins was evaluated by absorbance. The absorbance values were obtained by measuring the equivalent anthocyanin extract which were dissolved by phosphoric acid buffer solution. In the temperature range from $40^{\circ} \mathrm{C}$ to $100^{\circ} \mathrm{C}$,

taking 10 as the unit interval, six temperatures of $50^{\circ} \mathrm{C}, 60^{\circ} \mathrm{C}, 70^{\circ} \mathrm{C}, 80^{\circ} \mathrm{C}, 90^{\circ} \mathrm{C}$ and $10^{\circ} \mathrm{Cwere}$ selected and kept in the constant temperature water bath for $30 \mathrm{mi}$ $\mathrm{n}, 25 \mathrm{~min}, 20 \mathrm{~min}, 15 \mathrm{~min}, 10 \mathrm{~min}$ and $5 \mathrm{~min}$ respectively. The absorbance values were determined three times parallelly.

\subsection{Orthogonal experiment}

The orthogonal experimental factors were ultrasonic extraction temperature, ultrasonic extraction time and solid-liquid ratio. They are shown in Table 1.

Table1. Positive experimental factors

\begin{tabular}{cccc}
\hline $\begin{array}{c}\text { Level } \\
\text { determination } \\
\begin{array}{c}\text { Experimental } \\
\text { factors }\end{array}\end{array}$ & $\begin{array}{c}\text { (A) Time of } \\
\text { ultrasonic } \\
\text { extraction }\end{array}$ & $\begin{array}{c}\text { (B) Ultrasonic } \\
\text { extraction } \\
\text { temperature }\end{array}$ & $\begin{array}{c}\text { (C) Solid- } \\
\text { liquid ratio }\end{array}$ \\
\hline 1 & 20 & 30 & $1: 12.5$ \\
2 & 30 & 40 & $1: 15$ \\
3 & 40 & 50 & $1: 17.5$ \\
\hline
\end{tabular}

\section{Experimental Results and Analysis}

\subsection{Single-factor experiment}

\subsubsection{Time on extraction rate of anthocyanins}

The extraction time-absorbance curve is shown in figure 1(a) under the condition of the ethanol as extractant, the solid-liquid ratio of $1: 15$, the extraction temperature of $50^{\circ} \mathrm{C}$, and $10 \mathrm{~min}$ was selected as the unit time interval of 10-50 $\mathrm{min}$. The extraction rate gradually increased before the extraction time of $40 \mathrm{~min}$ and its maximum value appeared at $40 \mathrm{~min}$. Then, the extraction rate tended to be constant after $40 \mathrm{~min}$. The reason was that almost all anthocyanins in eggplant peel were extracted more than 40 min of extraction time, and the extraction rate remained unchanged. The extraction effect is the best at $40 \mathrm{~min}$ because short time will cause inadequate extraction, and long time will cause energy waste. 

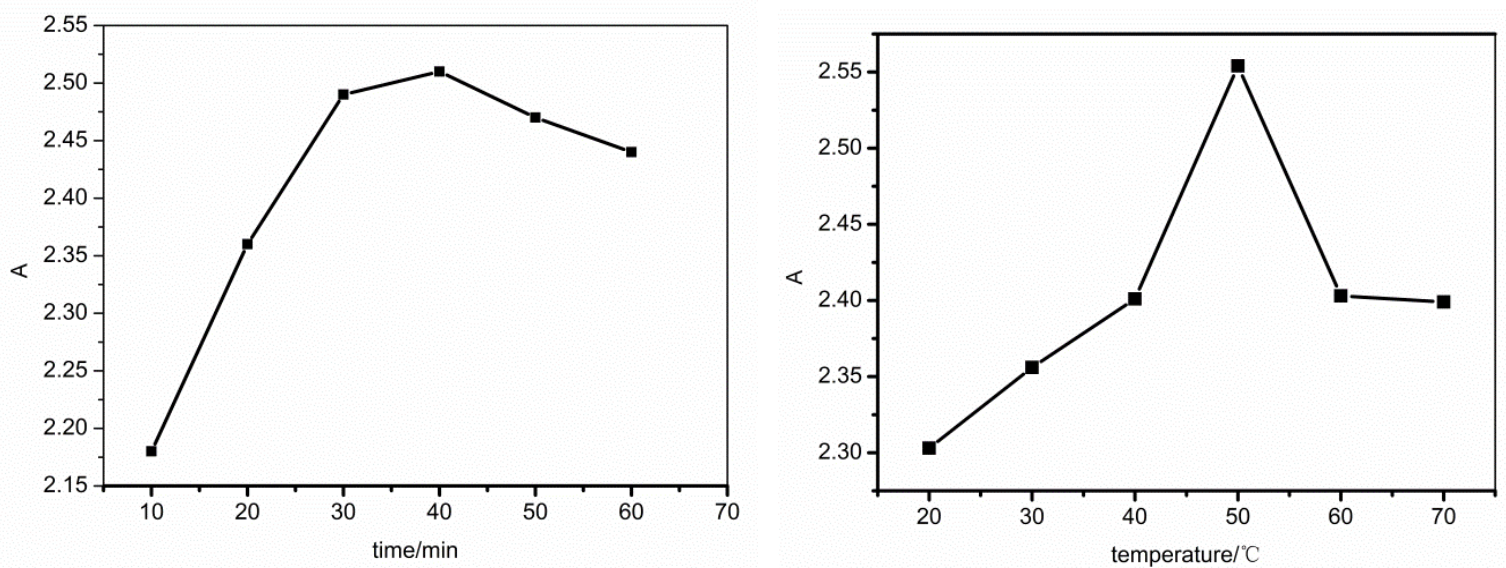

Fig 1. Effects of different factor on extraction rate of anthocyanins (a) different time (b) different temperature

\subsubsection{Temperature on extraction rate of anthocyanins}

The extraction temperature-absorbance curve is shown in figure 1(b) under the condition of the ethanol as extractant, the solid-liquid ratio of $1: 15$, and the extraction time of 40 min. According to the experimental data, the absorbance of the extract increases first and then decreases with the increase of temperature. The absorbance is the largest at $50^{\circ} \mathrm{C}$. It is speculated that if the temperature continues to rise, the absorbance will decrease. Therefore, increasing the temperature in a certain temperature range can increase the extraction rate of anthocyanin, but once it exceeds this range, the extraction rate will be significantly reduced. Therefore, the extraction rate of anthocyanin was the highest at $50^{\circ} \mathrm{C}$.

\subsubsection{Solid-liquid ratio on extraction rate of anthocyanins}

The solid-liquid ratio-absorbance curve is shown in figure 2 under the condition of the ethanol as extractant, the extraction temperature of $50^{\circ} \mathrm{C}$, and the extraction time of $40 \mathrm{~min}$. It can be seen from figure 2 that when the solidliquid ratio is below $1: 15$, the extraction rates increase and the absorbance of anthocyanins increases gradually. With the slow increase of the extraction solvent, anthocyanin and the extraction solvent can be fully contacted, increasing the amount of anthocyanin extraction, so the extraction rate increases. When the solid-liquid ratio exceeds $1: 15$, the absorbance decreases. So the best solidliquid ratio is $1: 15$.

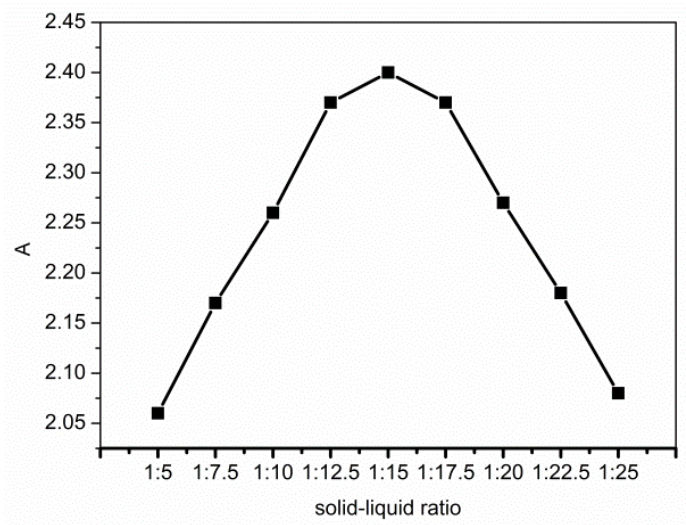

Fig 2. Effects of different solid-liquid ratio on extraction rate of anthocyanins

\subsection{Anthocyanin thermal stability test}

Anthocyanin solution of $\mathrm{pH}=4$ was prepared by citric acid-disodium hydrogen phosphate to examine the thermal stability of anthocyanin. They were heated in a water bath of $65^{\circ} \mathrm{C}$ for $30 \mathrm{~min}, 75^{\circ} \mathrm{C}$ for $15 \mathrm{~min}, 85^{\circ} \mathrm{C}$ for $10 \mathrm{~min}, 95^{\circ} \mathrm{C}$ for $5 \mathrm{~min}$, and $100^{\circ} \mathrm{C}$ for $1 \mathrm{~min}$, respectively. The absorbance was measured at $525 \mathrm{~nm}$. The results are shown in Table 2. According to the table, the absorbance of anthocyanin at $100^{\circ} \mathrm{C}$ is still larger than that at other temperatures, and the anthocyanin is stable at high temperature. So the anthocyanin can be stable in the production process of related products.

Table2. Thermal stability test results of anthocyanin

\begin{tabular}{ccc}
\hline Temperature $/{ }^{\circ} \mathrm{C}$ & Time/ min & $\begin{array}{c}\text { Absorbance } \\
\text { values }\end{array}$ \\
\hline 65 & 30 & 0.66 \\
75 & 15 & 0.52 \\
85 & 10 & 0.20 \\
95 & 5 & 0.47 \\
100 & 1 & 0.73 \\
\hline
\end{tabular}

\subsection{Orthogonal test}

Through the analysis of orthogonal software design, the 
orthogonal result can be obtained for different ultrasonic treatment time, ultrasonic treatment temperature and solid-liquid ratio, as shown in Table 3. Table 3 shows that the influence of various factors on anthocyanin extraction is $\mathrm{B}>\mathrm{C}>\mathrm{A}$. That is ultrasonic temperature $>$ solid-liquid ratio $>$ ultrasonic time. The optimal combination of extraction condition is $\mathrm{A}_{3} \mathrm{~B}_{3} \mathrm{C}_{2}$ that is solid-liquid ratio of $1: 15$, ultrasonic time of $40 \mathrm{~min}$, and ultrasonic temperature of $50^{\circ} \mathrm{C}$.

Table3. Orthogonal experimental results

\begin{tabular}{|c|c|c|c|c|}
\hline \multirow{2}{*}{$\begin{array}{c}\text { Experim } \\
\text { ental } \\
\text { serial } \\
\text { number }\end{array}$} & \multicolumn{3}{|c|}{ Factors } & \multirow{2}{*}{$\begin{array}{c}\text { Absor } \\
\text { bance/ } \\
\text { Anm } \\
\mathbf{5 2 5}\end{array}$} \\
\hline & $\begin{array}{c}\text { (A) } \\
\text { Ultrasonic } \\
\text { time }\end{array}$ & $\begin{array}{c}\text { (B) } \\
\text { Ultrasonic } \\
\text { temperature }\end{array}$ & $\begin{array}{l}\text { (C) } \\
\text { Solid- } \\
\text { liquid } \\
\text { ratio }\end{array}$ & \\
\hline 1 & 1 & 1 & 1 & 0.233 \\
\hline 2 & 2 & 1 & 2 & 0.269 \\
\hline 3 & 3 & 1 & 3 & 0.310 \\
\hline 4 & 1 & 2 & 2 & 0.245 \\
\hline 5 & 2 & 2 & 3 & 0.455 \\
\hline 6 & 3 & 2 & 1 & 0.332 \\
\hline 7 & 1 & 3 & 3 & 0.200 \\
\hline 8 & 2 & 3 & 1 & 0.306 \\
\hline 9 & 3 & 3 & 2 & 0.397 \\
\hline k1 & 0.226 & 0.272 & 0.290 & \\
\hline k2 & 0.343 & 0.259 & 0.304 & $\mathbf{A}_{3} \mathbf{B}_{3} \mathbf{C}_{2}$ \\
\hline k3 & 0.346 & 0.301 & 0.221 & \\
\hline
\end{tabular}

\section{Application of Anthocyanins in Cosmetics}

Studies have shown that anthocyanins have antioxidant, anti-mutation, and ascorbic acid-related physiological functions. They are a very healthy natural pigment of protecting the skin on the lips, resisting free radicals, reducing UV damage and melanin precipitation, preventing dark around the mouth, and maintaining proper elasticity and tension[7]. The principle that anthocyanins can show different colors in different acid and alkali environments which can be used to add them to cosmetics. In addition, anthocyanins can play a related physiological role to achieve the purpose of environmental protection and health.

\section{Conclusions}

Single factor experiment showed that ultrasonic time was a significant factor in the extraction of anthocyanin from eggplant peel. Through orthogonal experiment, the anthocyanin extracted from eggplant peel was the best under the condition of feed-liquid ratio of $1: 15$, time of 40 min, and temperature of $50^{\circ} \mathrm{C}$. The thermal stability of anthocyanin was good after $100^{\circ} \mathrm{C}$ for 1 min treatment. It shows that high temperature short-time treatment has little effect on the stability of anthocyanin. Anthocyanins of physiological functions can be used to add to cosmetics.

\section{References}

1. Yizhu Zhang, Yanyi Chen, Jianjun Chu. Effects of different extraction conditions on anthocyanin extraction rate of round eggplant [B], Biology Bulletin, 2014, 49: 50-53.

2. Yue Li, Liqiang Dong, Rui Ma. Extraction of anthocyan of anthocyanins from black bean peel in vitro [J]. Journal of Harbin Commercial University (Natural Science Edition), 2020, 36: 532-536.

3. Chunhui Wei, Lanlan Zhang, Jie Deng, et al. Optimization of ultrasonic assisted extraction process of mulberry anthocyanin,Food Industry, 2020, 41(12): 96-99.

4. Mengting Lu, Zhie Tao, Wenhui Qi, Fang Lin, Jie Pan. Studies on the extraction and thermal

5. stability of anthocyanin from the fruit skin of fire dragon fruit [J].Guang Zhou Chemical Industry, 2020, 48(23): 66-75.

6. Baoli Zhou, Qi Zhang, Xueling Ye, Zhixia Chen. Content and stability of anthocyanin in different varieties of eggplant peel [A]. Food Industry, 2011, 32: 99-103.

7. Li Tan, Ruizhan Chen, Yusha Peng, et al. Optimization of extraction process and evaluation of antioxidant activity of blueberry anthocyanin [J]. Food Industry, 2017, 38(8): 136-141.

8. Zheng Wang, Chao Wang, Linbo Zhang. Application of plant extracts in cosmetics [J]. Jilin Agriculture, 2011, 4: 318-319. 